\title{
The Newtonian Potential of the Static Scalar Particle
}

\author{
Yaoyang Liu*, Lazhen Sun \\ Department of Modern Physics, University of Science and Technology of China, Hefei, China
}

Email address:

liuyy1934@sina.com (Yaoyang Liu)

*Corresponding author

\section{To cite this article:}

Yaoyang Liu, Lazhen Sun. The Newtonian Potential of the Static Scalar Particle. American Journal of Modern Physics. Vol. 10, No. 2, 2021, pp. 36-40. doi: 10.11648/j.ajmp.20211002.13

Received: March 22, 2021; Accepted: April 12, 2021; Published: May 7, 2021

\begin{abstract}
In this article we obtain the Coulombian potential of spinor and scalar particle with the quantum field theory, there is no difference between the spinor and scalar particle. Similarly the Newtonian potential of spinor particle and scalar particle are also obtained with the quantum field theory, however it is found that the Newtonian potential of scalar particle is half of the Newtonian potential of spinor particle with same mass, it contradicts with the equality of gravitational and inertial mass. The Newtionian potential of the spinor particle can be used to obtain the Newtonian potentials of the sun,the earth and so on, therefore we suggest that it should be considered to be the experimental test of the gravitational theory of Einstein.
\end{abstract}

Keywords: Newtonian Potential, Gravitational, Inertial Mass, Tetrad Field, Coulombian Potential, Scalar Particle

\section{Introduction}

There are two interesting things to us, one is that the Coulombian potential used to treat the hydrogen bound state is introduced classically, so far, we have not had any attempt to get it by quantum field theory. The other thing is that the Newtonian potential is the logical inference of the classical Einstein theory, up to now we have not had any attempt to get it by quantum field theory. In this article we try firstly to obtain the Coulombian potential of the spinor and scalar particle with the quantum field theory, it is found that both give the same Coulomb potential with the same electric charge. Secondly we try to obtain the Newtonian potential of the spinor and scalar particle with the quantum field theory, it is found that the Newtonian potential of the scalar particle is half of the Newtonian potential of the spinor particle with same mass, obviously it contradicts with the equality of the gravitational and inertial mass.

Section 2 is devoted to the general formalism of $Q E D$ including one charged spinor and one scalar field[1]. In the quantum theory of hydrogen bound state the electron is bound by the proton with the classical Coulombian potential. How we can get the classical Coulomb potential from the of quantum field theory, we assume that the electromagnetic field appearing in the equation of motion of the electromagnetic field is a classical field but the material fields are taken to be quantum fields so the equation of motion of the electromagnetic field becomes a q-number equation. Furthermore at the weak-field approximation the material fields should be free fields, then one needs only the plane wave solutions of the material fields, especially the solution of the zero momentum is expressed in detail, also the $\delta(\mathbf{x})$ function is suggested to describe the point particle, finally the Coulombian potential of the static point charged spinor and scalar particle are obtained and there is no difference between spinor and scalar particle with the same electric charge.

Section 3 is devoted to the general formalism of the Einstein theory including tetrad, spinor and scalar field $[2,3,4,5,6]$ and also the Lagrange field which is necessary owing to the suggesting constraint $g^{\mu \nu}-E_{\alpha}^{\mu} E^{\nu}{ }_{\beta} \eta^{\alpha \beta}[7,8,9]$. In the Newton theory the Newtonian potential is taken to be a classical field which determine the movement of planets; in the Einstein theory the movement of the planets with lowvelocity is determined by the metric, then one can determine the metric from the Newton theory, that means this metric is not the direct inference of the Einstein theory $[5,7]$, the reason is of the lacking of a spinor field in the general formalism of Einstein theory[10,11,12] . Therefore we should firstly to get the equations of motion for various fields especially the equations of motion at the weak-field approximation. For the present aim of getting the Newtonian potential we need only 
the approximation equation of motion of the metric where the energy-momentum tensor is determined by the free spinor and scalar field as motioned in section 2 . By the same procedure the metric field is considered to be a classical field but the spinor and scalar field are quantum field then the Einstein equation is a q-number equation. We use the $\delta(\mathbf{x})$ function to describe the static point spinor and scalar particle then we obtain the Newtonian potential of the spinor and scalar particle but the Newtonian potential of the scalar particle is half of the Newtonian potential of spinor particle with the same mass. it contradicts with the equality of gravitational and inertial mass $[13]$.

\section{The Coulomb Potential for Spinor and Scalar Particle}

The action for the system including electromagnetic field, one spinor field, one complex scalar field is well known[1]

$$
I=-\int \frac{1}{4} F_{\mu \nu} F^{\mu \nu} d^{4} \mathbf{x}+\int \bar{\psi}\left[i \gamma^{\mu}\left(\partial_{\mu}-i e A_{\mu}\right)-M\right] \psi d^{4} \mathbf{x}+\int\left[\left(\left(\partial_{\mu}-i e A_{\mu}\right) \varphi\right)^{\dagger}\left(\partial^{\mu}-i e A^{\mu}\right) \varphi-m^{2} \varphi^{\dagger} \varphi\right] d^{4} \mathbf{x}
$$

from which we get the equations of motion, especially the equation of motion of the electromagnetic field

$$
\begin{aligned}
\partial_{\mu} F^{\mu \nu} & =e \bar{\psi} \gamma^{\nu} \psi \\
& +i e\left[\varphi^{\dagger}\left(\partial^{\nu}-i e A^{\nu}\right) \varphi-\left(\left(\partial^{\nu}-i e A^{\nu}\right) \varphi\right)^{\dagger} \varphi\right]
\end{aligned}
$$

By using the Lorentz gauge, in the weak-field approximation (2) becomes

$$
\square A^{\nu}=e \bar{\psi} \gamma^{\nu} \psi+i e\left[\varphi^{\dagger} \partial^{\nu} \varphi-\left(\partial^{\nu} \varphi\right)^{\dagger} \varphi\right] .
$$

In principle all the fields should be quantum fields but we want to obtain only the Coulombian potentials of the spinor particle and the scalar particle, we will consider $A^{\nu}$ being classical field and $\psi, \varphi$ being free quantum fields. The Fourier plane wave series of the free spinor and complex scalar field are

$$
\begin{aligned}
\psi(\mathbf{x}, t) & =\sum_{s_{ \pm}} \sum_{\mathbf{p}} \frac{1}{\sqrt{L^{3}}} \sqrt{\frac{M}{E_{p}}}\left[b_{\mathbf{p} s} u(\mathbf{p}, s) e^{-i p x}+d_{\mathbf{p} s}^{\dagger} v(\mathbf{p}, s) e^{i p x}\right] \\
\bar{\psi}(\mathbf{x}, t) & =\sum_{s_{ \pm}} \sum_{\mathbf{p}} \frac{1}{\sqrt{L^{3}}} \sqrt{\frac{M}{E_{p}}}\left[b_{\mathbf{p} s}^{\dagger} \bar{u}(\mathbf{p}, s) e^{i p x}+d_{\mathbf{p} s} \bar{v}(\mathbf{p}, s) e^{-i p x}\right] \\
\varphi(\mathbf{x}, t) & =\sum_{\mathbf{p}} \frac{1}{\sqrt{2 \omega_{p} L^{3}}}\left[a_{+\mathbf{p}} e^{-i p x}+a_{-\mathbf{p}}^{\dagger} e^{i p x}\right] \\
\varphi^{\dagger}(\mathbf{x}, t) & =\sum_{\mathbf{p}} \frac{1}{\sqrt{2 \omega_{p} L^{3}}}\left[a_{+\mathbf{p}}^{\dagger} e^{i p x}+a_{-\mathbf{p}} e^{-i p x}\right]
\end{aligned}
$$

where we suppose that the variable region of $\mathrm{x}$ is a box with each side $L$, then the periodic boundary condition requires that the momentum $\mathbf{p}$ will be

$$
p_{i}=\frac{2 \pi n_{i}}{L}, \quad n_{i}=\text { integer }, \quad s= \pm \frac{1}{2}, E_{P}=\sqrt{P^{2}+M^{2}}, \quad \omega_{P}=\sqrt{P^{2}+m^{2}},
$$

here $b_{\mathbf{p} s}, d_{\mathbf{p} s}, a_{+\mathbf{P}}, a_{-\mathbf{P}}$ and $b_{\mathbf{p} s}^{\dagger}, d_{\mathbf{p} s}^{\dagger}, a_{+\mathbf{P}}^{\dagger}, a_{-\mathbf{P}}^{\dagger}$ are operators satisfying the usual commutation relations.

Our purpose is to get the Coulombian potential for static particle so $\psi, \varphi$ in $(3)$ should be replaced by

$$
\begin{aligned}
\psi(\mathbf{x}, t) & \rightarrow \sum_{s_{ \pm}} \frac{1}{\sqrt{L^{3}}}\left[b_{\mathbf{0} s} u(\mathbf{0}, s) e^{-i m t}+d_{\mathbf{0} s}^{\dagger} v(\mathbf{0}, s) e^{i m t}\right] \\
\bar{\psi}(\mathbf{x}, t) & \rightarrow \sum_{s_{ \pm}} \frac{1}{\sqrt{L^{3}}}\left[b_{\mathbf{0} s}^{\dagger} \bar{u}(\mathbf{0}, s) e^{i m t}+d_{\mathbf{0} s} \bar{v}(\mathbf{0}, s) e^{-i m t}\right] \\
\varphi(\mathbf{x}, t) & \rightarrow \frac{1}{\sqrt{2 m L^{3}}}\left[a_{+\mathbf{0}} e^{-i m t}+a_{-\mathbf{0}}^{\dagger} e^{i m t}\right] \\
\varphi^{\dagger}(\mathbf{x}, t) & \rightarrow \frac{1}{\sqrt{2 m L^{3}}}\left[a_{+\mathbf{0}}^{\dagger} e^{i m t}+a_{-\mathbf{0}} e^{-i m t}\right]
\end{aligned}
$$


The $c$-number relation (3) will be obtained by the matrix elements of single static particles, these include the single static particle $\left|1_{b 0 s}\right\rangle$, single static anti-particle $\left|1_{d 0 s}\right\rangle$, single static positive charge boson $\left|1_{+0}\right\rangle$, single static negative charge boson $\left|1_{-0}\right\rangle$, it is easy to realize that $A^{i}=0$, we have

$$
\begin{aligned}
\square A^{0} & =<1_{b 0 s}\left|: e \sum_{s_{ \pm}} \frac{1}{\sqrt{L^{3}}}\left[b_{0 s}^{\dagger} \bar{u}(\mathbf{0}, s) e^{i m t}+d_{0 s} \bar{v}(\mathbf{0}, s) e^{-i m t}\right] \gamma^{0} \times \sum_{s_{ \pm}} \frac{1}{\sqrt{L^{3}}}\left[b_{0 s} u(\mathbf{0}, s) e^{-i m t}+d_{0 s}^{\dagger} v(\mathbf{0}, s) e^{i m t}\right]:\right| 1_{b 0 s}> \\
& =e \frac{1}{L^{3}} \bar{u}(\mathbf{0}, s) \gamma^{0} u(\mathbf{0}, s)=e \frac{1}{L^{3}} u^{\dagger}(\mathbf{0}, s) u(\mathbf{0}, s)=e \frac{1}{L^{3}}
\end{aligned}
$$

where $e \frac{1}{L^{3}}$ is the density of the electric charge, we suppose that the corresponding expression of static point particle at the origin can be obtained by the substitution

$$
e \frac{1}{L^{3}} \rightarrow e \delta(\mathbf{x})
$$

One gets

$$
\triangle A^{0}=-e \delta(\mathbf{x}), \quad A^{0}=\frac{e}{4 \pi r} .
$$

Similarly for the static point anti-particle

$$
\triangle A^{0}=e \delta(\mathbf{x}), \quad A^{0}=-\frac{e}{4 \pi r} .
$$

For the single positive charged boson, one obtains

$$
\square A^{0}=i e \frac{1}{2 m L^{3}}<1_{+0}\left|:\left(a_{+0}^{\dagger} e^{i m t}+a_{-0} e^{-i m t}\right) \partial^{0}\left(a_{+0} e^{-i m t}+a_{-0}^{\dagger} e^{i m t}\right) \varphi-h . c .:\right| 1_{+0}>=e \frac{1}{L^{3}},
$$

for the static point positive charged boson, by using the substitution (14) we have

$$
\triangle A^{0}=-e \delta(\mathbf{x}), \quad A^{0}=\frac{e}{4 \pi r} .
$$

Similarly for the static point negative charged boson we have

$$
\triangle A^{0}=e \delta(\mathbf{x}), \quad A^{0}=-\frac{e}{4 \pi r} .
$$

One sees that there is no difference in electric properties between the charged spinor particle and the charged boson particle.

\section{The Newtonian Potential for Spinor and Scalar Particle}

The action for the system including gravitational field, one spinor field, one complex scalar field in the tetrad formalism is [2-6]

$$
\begin{aligned}
I & =\frac{1}{16 \pi G} \int R \sqrt{-g} d^{4} \mathbf{x}+\int \bar{\psi}\left(i \gamma^{\mu} D_{\mu}-M\right) \psi \sqrt{-g} d^{4} \mathbf{x} \\
& +\int \lambda^{\sigma \rho}\left(g_{\sigma \rho}-g_{\sigma \mu} g_{\rho \nu} \bar{E}^{\mu} E^{\nu}\right) \sqrt{-g} d^{4} \mathbf{x} \\
& +\int\left[g^{\mu \nu} \frac{\partial \varphi^{\dagger}}{\partial x^{\mu}} \frac{\partial \varphi}{\partial x^{\nu}}-m^{2} \varphi^{\dagger} \varphi\right] \sqrt{-g} d^{4} \mathbf{x} .
\end{aligned}
$$

Where we assume that for every space-time point $\mathbf{x}$ there is a tangent space of Hilbert space, or Lorentz space simply, in which one can define scalar field $\varphi$, spinor field $\psi$, furthermore for each direction $\mu$ of the Riemann space, there are four tetrad fields $\mathbf{E}^{\mu}(\mathbf{x})$ of the Lorentz space with four components $E_{\alpha}^{\mu}$, the four vectors are also contravariant vectors under the general coordinate transformation. We choose the latter Greek alphabets $\mu, \nu, \cdots$ to denote the index of the left column of $E_{\alpha}^{\mu}$, their raising and lowing are with $g^{\mu \nu}$ and choose the former Greek alphabets $\alpha, \beta, \cdots$ to denote the index of the right column of $E_{\alpha}^{\mu}$ their raising and lowing are with $\eta^{\alpha \beta}$, we use the convention $\operatorname{dig} \eta^{\alpha \beta}=(1,-1,-1,-1)$. By the tetrad fields one can obtain the the covariant derivative of the spinor field under the locally Lorentz transformation then one can obtain the action of spinor field to be invariance under both the Lorentz transformation and the general coordinate transformation. In addition we suppose a constraint $g^{\mu \nu}-$ $E_{\alpha}^{\mu} E^{\nu}{ }_{\beta} \eta^{\alpha \beta}$ and the corresponding Lagrange multiplier field $\lambda^{\sigma \rho}$.

$$
\begin{aligned}
& \gamma^{\mu}=E_{\alpha}^{\mu} \gamma^{\alpha} \\
& D_{\mu} \psi=\left(\partial_{\mu}+A_{\mu}\right) \psi \\
& A_{\mu}=\frac{1}{2} \sigma^{\alpha \beta} E_{\alpha}^{\sigma} \partial_{\mu} E_{\sigma \beta}=\frac{1}{2} \sigma^{\alpha \beta} g_{\sigma \rho} E_{\alpha}^{\sigma} \partial_{\mu} E_{\beta}^{\rho}, \\
& \sigma^{\alpha \beta}=\frac{1}{4}\left[\gamma^{\alpha}, \gamma^{\beta}\right] \\
& {\left[\sigma^{\alpha \beta}, \sigma^{\gamma \delta}\right]=\eta^{\beta \gamma} \sigma^{\alpha \delta}-\eta^{\alpha \gamma} \sigma^{\beta \delta}-\eta^{\delta \alpha} \sigma^{\gamma \beta}+\eta^{\delta \beta} \sigma^{\gamma \alpha}} \\
& \left(\omega^{\alpha \beta}\right)_{\gamma}^{\varepsilon}=\left(\delta_{\gamma}^{\alpha} \delta_{\epsilon}^{\beta}-\delta_{\epsilon}^{\alpha} \delta_{\gamma}^{\beta}\right) \eta^{\epsilon \varepsilon}
\end{aligned}
$$




$$
\left[\omega^{\alpha \beta}, \omega^{\gamma \delta}\right]=\eta^{\beta \gamma} \omega^{\alpha \delta}-\eta^{\alpha \gamma} \omega^{\beta \delta}-\eta^{\delta \alpha} \omega^{\gamma \beta}+\eta^{\delta \beta} \omega^{\gamma \alpha}
$$

By (20) one obtains the equations of motion for $\psi, \varphi, \lambda^{\sigma \rho}$, especially the Einstein equation

$$
R_{\mu \nu}-\frac{1}{2} g_{\mu \nu} R=-8 \pi G T_{\mu \nu}
$$

Up to now we have no a quantum theory of gravitation so we consider the gravitational field to be classical field, but the fields $\psi, \varphi$ are quantum fields. In the weak-field approximation

$$
\begin{aligned}
& E_{\alpha}^{\mu}=\eta_{\alpha}^{\mu}+e_{\alpha}^{\mu}, \quad\left|e_{\alpha}^{\mu}\right|<<1, \\
& g_{\mu \nu}=\eta_{\mu \nu}-s_{\mu \nu}, \quad\left|s_{\mu \nu}\right|<<1, \\
& s_{\mu \nu}=e_{\mu \nu}+e_{\nu \mu},
\end{aligned}
$$

we have obtained the approximate energy-momentum tensor of spinor field [6], and now the approximate energy-momentum tensor of scalar field can be obtained easy, so the approximate Einstein equation is

$$
\begin{aligned}
\square s_{\mu \nu} & =16 \pi G\left(T_{\mu \nu}-\frac{1}{2} \eta_{\mu \nu} T_{\lambda}^{\lambda}\right), \\
T_{\mu \nu} & =: \frac{1}{2} \bar{\psi} i\left(\gamma_{\mu} \partial_{\nu}+\gamma_{\nu} \partial_{\mu}\right) \psi:+: \frac{1}{2}\left(\partial_{\mu} \varphi^{\dagger} \partial_{\nu} \varphi+\partial_{\nu} \varphi^{\dagger} \partial_{\mu} \varphi\right)-\frac{1}{2}\left[\left(\partial_{\lambda} \varphi^{\dagger}\right)\left(\partial^{\lambda} \varphi\right)-m^{2} \varphi^{\dagger} \varphi\right] \eta_{\mu \nu}: .
\end{aligned}
$$

Because $\psi, \varphi$ are quantum fields, a classical equation is a matrix elements between quantum states, our purpose is to get the Newtonian potential of the static spinor particle and static scalar particle then we need only the terms $(9),(10),(11),(12)$.

For the static single spinor particle one can see that the only non-zero matrix element is

$$
\begin{aligned}
<1_{b 0 s}\left|T_{00}\right| 1_{b 0 s}> & =<1_{b 0 s}\left|\frac{1}{2} \bar{\psi} i\left(\gamma_{0} \partial_{0}+\gamma_{0} \partial_{0}\right) \psi\right| 1_{b 0 s}> \\
& =\frac{1}{L^{3}}<1_{b 0 s}\left|:\left[b_{0 s}^{\dagger} \bar{u}(\mathbf{0}, s) e^{i M t}+d_{0 s} \bar{v}(\mathbf{0}, s) e^{-i M t}\right] i \gamma_{0} \partial_{0}\left[b_{0 s} u(\mathbf{0}, s) e^{-i M t}+d_{0 s}^{\dagger} v(\mathbf{0}, s) e^{i M t}\right]:\right| 1_{b 0 s}> \\
& =\frac{1}{L^{3}} M \bar{u}(\mathbf{0}, s) \gamma_{0} u(\mathbf{0}, s)=\frac{1}{L^{3}} M u^{\dagger}(\mathbf{0}, s) u(\mathbf{0}, s)=\frac{M}{L^{3}}
\end{aligned}
$$

As mentioned above, $M \frac{1}{L^{3}}$ is the density of the energy-momentum tensor, we suppose that the corresponding expression of static point particle at the origin can be obtained by the substitution (14).

$$
\begin{gathered}
<1_{b 0 s}\left|T_{00}\right| 1_{b 0 s}>=M \delta(\mathbf{x}), \\
\triangle s_{00}=\triangle s_{i i}=-8 \pi M \delta(\mathbf{x}), \quad \triangle s_{0 i}=\triangle s_{i 0}=0, \\
s_{00}=s_{i i}=\frac{2 M G}{r}, \quad s_{0 i}=s_{i 0}=0 .
\end{gathered}
$$

For the static single single anti-particle, it is easy to have

$$
<1_{d 0 s}\left|T_{00}\right| 1_{d 0 s}>=M \delta(\mathbf{x})
$$

so the static single single anti-particle give the same Newtonian potential.

For the single positive static boson particle and the single negative static boson particle we have

$$
\begin{aligned}
& <1_{+0}\left|T_{00}\right| 1_{+0}>=\frac{1}{4 m L^{3}}<1_{+0}\left|i e:\left[\partial_{0}\left(a_{+0}^{\dagger} e^{i m t}+a_{-0} e^{-i m t}\right) \times \partial_{0}\left(a_{+0} e^{-i m t}+a_{-0}^{\dagger} e^{i m t}\right)+h . c .\right]\right| 1_{+0}>=\frac{m}{2 L^{3}}, \\
& <1_{-0}\left|T_{00}\right| 1_{-0}>=\frac{1}{4 m L^{3}}<1_{-0}\left|i e:\left[\partial_{0}\left(a_{+0}^{\dagger} e^{i m t}+a_{-0} e^{-i m t}\right) \times \partial_{0}\left(a_{+0} e^{-i m t}+a_{-0}^{\dagger} e^{i m t}\right)+h . c .\right]\right| 1_{-0}>=\frac{m}{2 L^{3}},
\end{aligned}
$$

for the static point boson particle we have

$$
\begin{gathered}
<1_{+0}\left|T_{00}\right| 1_{+0}>=<1_{-0}\left|T_{00}\right| 1_{-0}>=\frac{m}{2} \delta(\mathbf{x}), \\
\triangle s_{00}=\triangle s_{i i}=-4 \pi m G \delta(\mathbf{x}), \quad \triangle s_{0 i}=\triangle s_{i 0}=0,
\end{gathered}
$$




$$
s_{00}=s_{i i}=\frac{m G}{r}, \quad s_{0 i}=s_{i 0}=0 .
$$

So we can conclude that the Newtonian potential of the static point boson particle is half of the Newtonian potential of the static point spinor particle with the same mass. It can be also proved that the relations of (42)(43) are applicable to the case of neutral boson particle.

\section{Discussion}

In the tetrad formalism the gravitational interaction is described by sixteen fields $E_{\alpha}^{\mu}$, its meaning can be recognized more clearly in the weak-field approximation, where the metric $g_{\mu \nu}$ are determined by the symmetric part of $e_{\mu \nu}$ and the $A_{\mu}$ are determine by the anti-symmetric part of $e_{\mu \nu}$.

$$
\begin{aligned}
A_{\mu} & =\frac{1}{2} \sigma^{\alpha \beta} g_{\sigma \rho} E_{\alpha}^{\sigma} \partial_{\mu} E_{\beta}^{\rho} \cong \frac{1}{4} \sigma^{\alpha \beta} \partial_{\mu} a_{\alpha \beta}, \\
a_{\alpha \beta} & =e_{\alpha \beta}-e_{\beta \alpha} .
\end{aligned}
$$

one gets the equation of motion of the spinor field approximately

$$
\begin{gathered}
\left(i \gamma^{\mu} \partial_{\mu}-M\right) \psi+i \frac{1}{4} \gamma^{\delta} \sigma^{\alpha \beta} \partial_{\delta} a_{\alpha \beta} \psi \cong 0 \\
\gamma^{\mu}=\left(\eta^{\mu \alpha}+\frac{1}{2} s^{\mu \alpha}+\frac{1}{2} a^{\mu \alpha}\right) \gamma_{\alpha} .
\end{gathered}
$$

One can see clearly that the gravitational effects of spin is of the small higher effect, although it is very difficult to treat theoretically and experimentally but its existence is not unacceptable.

The introducing of constraint $g^{\mu \nu}-E_{\alpha}^{\mu} E^{\nu} \eta^{\alpha \beta}$ seems by hands because in the Lagrangian form of the usual gauge theory the constraint appears naturally, however in the Hamiltonian formalism of the usual gauge theory the constraint is look like also as by hands. So we think this question is not a fundamental problem, the more importance is about the self-consistence of this constraint, in other words in order to maintein the constraint one should find next constraints and so on, or this constraint is incorrect by the Hamiltonian equation $[8,9]$. Unfortunately up to now we have no a theory of quantum gravitational field, so we assume that $g^{\mu \nu}-E_{\alpha}^{\mu} E_{\beta}^{\nu} \eta^{\alpha \beta}$ is the only constraint.

\section{Conclusion}

Firstly the Coulombian potential of the boson and spinor particle are obtained by quantum field theory, and also the
Coulombian potential of the proton is already known, so this calculation may be used the check the reliability of our computing method. Secondly the Newtonian potential of the spinor particle have been used to get the Newtonian potential of our solar system $[5,6,7]$, so the Newtonian potential of the spinor particle may be considered to be the experimental test of Einstein theory [6]. Thirdly the Newtonian potential of scalar particle is half of the Newtonian potential of spinor particle with the same mass, it contradicts the classical concept. This result reminds us that the invariance of the general coordinate transformation is the fundamental principle, in some cases the quantum theory give us a very different physical picture as one met in the Dirac theory of electron. We expect to have the experimental test although it is very difficult.

\section{References}

[1] J. D. Bjorken and S. D. Drell, Relativistic Quantum Mechanics, McGraw-Hill Book, 7 Company, (1964); Relativistic Quantum Fields, McGraw-Hill Book Company, (1965).

[2] C. N. Yang, R. L. Mills, Phys. Rev. 96, 191 (1954).

[3] R. Utiyama, Phys. Rev., 101, 1597 (1956).

[4] T. W. B. Kibble, J. Math. Phys., 2, 212 (1961).

[5] S. Weinberg, Gravitation and Cosmology, John Wiley, New York, (1972).

[6] Yao-Yang Liu and La-Zhen Sun, Mod. Phys. Lett. A, 35. No 34, 2050286(2020);https://doi.org/ $10.1142 / \mathrm{S} 0217732320502867$.

[7] P. A. M. Dirac, General Theory of Relativity, John Wiley, (1975)

[8] P. A. M. Dirac, Lectures on Quantum Mechanics, New York, Yeshiva University, (1964).

[9] L. D. Fadeev, V. N. Popov, Phys. Lett., bf B25, 30 (1967).

[10] D. Burns and A. Pilatsis, Phys. Rew., D91, (6) (2014).

[11] J. Edholm and A. Conroy, Phys. Rev. D96 4 (2017).

[12] M. L. Ruggiero and L. Lorio, J. of Cosmology and Astroparticle Phys., 6042 (2020)

[13] R. v. Eötvös, Math. Nat. Ber. Ungarn 865 (1890). 